Respiration 2014;88:518

DOI: $10.1159 / 000367813$

\section{M-Mode: A Valuable Tool in Cardiology, Is Not Yet Ready to Use in Pneumology}

Guglielmo M. Trovato ${ }^{\mathrm{a}}$, Daniela Catalano ${ }^{\mathrm{a}}$, Marco Sperandeo ${ }^{\mathrm{b}}$

a Department of Internal Medicine, AOU Policlinico VE, University of Catania, Catania, and b Department of Internal

Medicine, IRCCS Ospedale Casa Sollievo della Sofferenza,

San Giovanni Rotondo, Italy

We read with great interest the article by Smargiassi et al. [1] which elegantly presents a novel morphological-dynamic method using M-mode diaphragm and thoracic ultrasound (TUS) clinical assessment in chronic obstructive pulmonary disease (COPD). They appropriately state that the role of TUS is valuable as a complementary noninvasive technique in the assessment of a variety of pulmonary conditions, which is also our experience in 25 years of clinical practice. TUS is a very important imaging tool since the objects of its study are very prevalent, severe and, hopefully, treatable, involving diseases such as pneumonia, pneumothorax, serous effusions, fibrosis autoimmune disease and lung cancer [2]. It is correct, in our view, not to rely inappropriately on artifacts for anatomical and functional measurements $[3,4]$. Overall, the added value of TUS is valuable for reaching early, tentative preliminary diagnosis; more important is the fact that TUS allows a safe interventional guidance of cavity evacuations or of fine-needle aspiration biopsy procedures under the continuous visual control of the operator [5-8].

Smargiassi et al. [1] aim to investigate, in a sample of COPD patients, the ultrasound features of the diaphragm (i.e. thickness, thickening and excursions) in relation to both body composition (in terms of fat-free mass, FFM) and pulmonary function. Below, we will consider a few, although not all, key points.

Firstly, echographic assessment of the thickness of the diaphragm (TD) was done in the zone of apposition (ZOA) at various lung volumes, i.e. TD at residual volume (TDRV), TD at functional residual capacity (TDFRC) and TD at total lung capacity (TDTLC), which measured 3.3, 3.6 and $6 \mathrm{~mm}$, respectively, with good intraobserver reproducibility. However, a degree of oversimplification is present in the article [1]. The TD measurements were made at the end of a normal expiration (corresponding to functional residual capacity), during a breath-holding maneuver after maximal inspiration (corresponding to total lung capacity) and at the end of maximal expiration (corresponding to residual volume), and they were reported as TDFRC, TDTLC and TDRV, respectively. The differences between these three measurements were reported as diaphragm thickenings. The main problem is that no information is provided in regard to the actual reliability resolution of the millimeter measurements by M-mode when such tiny differences are to be detected. Fair inter- and intraobserver analysis are not a sufficient answer to this limitation. In other words, is it so easy to decide between a measurement of 3.0 and $4.0 \mathrm{~mm}$, since this is the size of difference and change?
Secondly, the interpretation of the body composition relationship with the M-mode measurements of the diaphragm and with pulmonary function tests is not convincing. The BMI in the studied population was $27.6 \pm 5.2$. This distribution means that some subjects had a BMI above 30.0, or even 35.0, whilst others could be underweight. Also, obesity and not only muscle wasting has an effect on pulmonary function, although this is not evident from the presented results. We believe this aspect deserves some comment. The interpretation that the relationship between TD and FFM 'was found to be the main determinant of TD' could be misleading, since in their results the same level of correlation was found with BMI as with TD (all p > 0.01).

Thirdly, the ZOA is probably not familiar to every reader, i.e. the area of the diaphragm encompassing the cylindrical portion (the part of the muscle shaped like a dome/umbrella) which corresponds to the portion directly apposed to the inner aspect of the lower rib cage. We would encourage the authors to comment on the fact that if the ZOA is decreased or suboptimal, there are several potential negative consequences in respiratory function, and, conceivably, in the quality of the images.

M-mode is still a greatly valuable, even neglected, method in cardiology, relying on the quality of images and measurements after accurate validation studies. We appreciate the efforts of Smargiassi et al. [1] which challenge the possibility of relying on objective imaging achieved by lung ultrasound, such as is already done for pleural effusion, and on quantitative, objective and reproducible measurements, such as in echocardiography. Nonetheless, the evidence provided is, in our view, insufficient and confirms that TUS is not the easiest of techniques to master and interpret. The inherent risk of generating misleading or inappropriate information may be exacerbated by overconfidence and reliance on artifacts, and on measurements derived by tools with a low degree of resolution.

\section{References}

1 Smargiassi A, Inchingolo R, Tagliaboschi L, Di Marco Berardino A, Valente S, Corbo GM: Ultrasonographic assessment of the diaphragm in chronic obstructive pulmonary disease patients: relationships with pulmonary function and the influence of body composition - a pilot study. Respiration 2014;87:364-371.

- 2 Trovato GM, Sperandeo M: Sounds, ultrasounds, and artifacts: which clinical role for lung imaging? Am J Respir Crit Care Med 2013;187: 780-781.

- 3 Trovato GM, Catalano D, Martines GF, Sperandeo M: Is it time to measure lung water by ultrasound? Intensive Care Med 2013;39:1662.

4 Trovato GM, Rollo VC, Martines GF, Catalano D, Trovato FM, Sperandeo M: Thoracic ultrasound in the differential diagnosis of severe dyspnea: a reappraisal. Int J Cardiol 2013;167:1081-1083.

5 Trovato GM, Sperandeo M, Catalano D: Optimization of thoracic US guidance for lung nodule biopsy. Radiology 2014;270:308.

6 Sperandeo M, Trovato GM, Dimitri L: Ultrasound elastography pattern of lung squamous cell carcinoma: preliminary report on a possible adjunctive tool for noninvasive imaging. J Clin Oncol 2013;31:1851-1858.

7 Trovato GM, Sperandeo M, Catalano D: Computed tomography screening for lung cancer. Ann Intern Med 2013;159:155.

8 Trovato GM, Sperandeo M, Catalano D: Thoracic ultrasound guidance for access to pleural, peritoneal, and pericardial space. Chest 2013;144: $1735-1736$

\section{KARGER}

E-Mail karger@karger.com

www.karger.com/res
2014 S. Karger AG, Basel

0025-7931/14/0886-0518\$39.50/0
Guglielmo M. Trovato, MD

Department of Medical and Pediatric Sciences, University of Catania via Santa Sofia, Policlinico Edificio 4

IT-95100 Catania (Italy)

E-Mail guglielmotrovato@unict.it 